\title{
ROLE OF MRI IN EVALUATION OF SEIZURES
}

\author{
Avadhesh Pratap Singh Kushwah1, Ketki Kedar², Sonjjay Pande ${ }^{3}$
}

${ }^{1}$ Assistant Professor, Department of Radiodiagnosis, NSCB Medical College, Jabalpur, Madhya Pradesh, India.

2 Post Graduate Student, Department of Radiodiagnosis, NSCB Medical College, Jabalpur, Madhya Pradesh, India.

3Professor \& HOD, Department of Radiodiagnosis, NSCB Medical College, Jabalpur, Madhya Pradesh, India.

ABSTRACT
BACKGROUND
Seizure is a paroxysmal alteration in neurologic function resulting from abnormal excessive neuronal electrical activity. Epilepsy
is a chronic condition characterized by recurrent seizures unprovoked by an acute systemic or neurologic insult.1 An epileptic seizure
is a clinical manifestation of abnormal, excessive neuronal activity arising in the grey matter of the cerebral cortex.

\section{MATERIALS AND METHODS}

Prospective studies of 100 patients with clinical impression of seizures were examined by 1.5 Tesla magnetic resonance imaging.

\section{RESULT}

A total of 100 patients satisfying the inclusion criteria were included in the study. The age range of patients was from neonate to elderly with male predominance, male $64(64 \%)$ and female $36(36 \%)$. GTCS was the most common clinical diagnosis constituting $(80 \%)$ cases. The common abnormalities were cerebral infarction with gliosis (16\%), infections - NCC (7\%) and tuberculoma (10\%), cerebral atrophy (1\%), developmental cortical malformations (2\%), venous thrombosis (4\%), low-grade glioma (9\%), meningioma (3\%).

\section{CONCLUSION}

MRI is the investigation of choice in patients with seizure disorder. The sensitivity of MRI in detecting abnormalities in patients with seizure disorder is in part associated to the underlying pathologies and by the MRI techniques and experience of the interpreting physician. Accurate diagnosis of the cause of seizure is crucial for finding an effective treatment. With its high spatial resolution, excellent inherent soft tissue contrast, multiplanar imaging capability and lack of ionizing radiation, MR imaging has emerge d as a versatile tool in the evaluation of patients with seizure disorder.

\section{KEYWORDS}

Epilepsy, MRI, Seizure.

HOW TO CITE THIS ARTICLE: Kushwah APS, Kedar K, Pande S. "Role of MRI in evaluation of seizures." Journal of Evolution of Medical and Dental Sciences 2015; Vol. 4, Issue 105, December 31; Page: 16977-16983, DOI: 10.14260/jemds/2015/2564

\section{INTRODUCTION}

Seizure is a paroxysmal alteration in neurologic function resulting from abnormal excessive neuronal electrical activity. Epilepsy is a chronic condition characterized by recurrent seizures unprovoked by an acute systemic or neurologic insult. ${ }^{1}$ An epileptic seizure is a clinical manifestation of abnormal, excessive neuronal activity arising in the grey matter of the cerebral cortex. Up to $10 \%$ of population will have at least one seizure in their lifetime, but only $2 \%$ of the population will develop epilepsy. Approximately, 34 to 76 new cases per 100,000 diagnosed every year. ${ }^{2}$

In order to diagnose and find out the etiology of the lesion, there are many neuro-radiological investigations that can be utilized. These include x-ray of skull, pneumocephalography, CSF examination, carotid angiography, EEG, CT and MRI. In this context, the revolutionary introduction of MRI for evaluation of seizures has been a great boon, both for the diagnosis of cerebral lesions as well as clinical management of patients with neurologic disorders.

Financial or Other, Competing Interest: None.

Submission 20-12-2015, Peer Review 21-12-2015,

Acceptance 26-12-2015, Published 29-12-2015.

Corresponding Author:

Dr. Avadhesh Pratap Singh Kushwah,

Plot No-137, PNT Colony, Dhanvantri Nagar,

Jabalpur-482003, Madhya Pradesh, India

E-mail: kushwahavadhesh@yahoo.com

DOI:10.14260/jemds/2015/2564
The superiority of Magnetic Resonance Imaging (MRI) over X-ray, Computed Tomography (CT) scanning in terms of sensitivity and specificity for identifying the aetiology of epilepsy in both adults and children is firmly established. The most common abnormalities identified are Hippocampal Sclerosis (HS), Malformations of Cortical Development (MCD), vascular malformations, tumours and acquired cortical damage.

The principal clinical applications of MRI are to identify the structural basis of epilepsy and patients, who are suitable for surgical treatment. Rapid advances are being made in MRI techniques, so that patients who were previously regarded as being MRI negative may have relevant abnormalities, which can be identified with contemporary optimal imaging. MR imaging has emerged as the more diagnostically relevant and most valuable tool for preoperative localization of epileptogenic focus because of its excellent soft tissue contrast, allowing for detailed depiction of anatomy, freedom from beam-hardening artifact in basal brain that occur with CT and capacity for multiplanar imaging. ${ }^{1}$

The diagnosis of epilepsy with the help of MRI has made this diagnostic tool beyond compare to other investigations. Postoperative MR may detect reasons for failure such as inadequate resection and can monitor tumour recurrence on follow up imaging. MRI is especially useful for prognosticating postoperative seizure control. ${ }^{1}$ 
The following study explains the role of MRI in depicting the etiology and spectrum of MRI findings in patients with seizure disorder.

\section{MATERIAL AND METHODS}

Prospective studies of 100 patients with clinical impression of seizures from November 2014 to October 2015 were subjected to MRI scanning.

\section{Source of Data:}

- NSCBMCH - Netaji Subhash Chandra Bose Medical College and Hospital, Jabalpur.

\section{Inclusion Criteria}

- All patients presented with seizures.

- Cases were included irrespective of age/sex.

\section{Exclusion Criteria}

- Contraindications to MRI studies.

\section{Statistical Analysis}

Quantitative variables expressed by using proportions and percentage and other statistical tools.

\section{Criteria for Patient's Selection}

- A detailed history was taken and clinical examination was done. The points noted were duration of illness, type of seizures, any associated illness. Detailed clinical and neurological examination was done to find any neurological deficit. Based on the history and examination, a clinic etiological diagnosis was made.

- Follow-up of cases was done until the completion of treatment (Maximum 6 months).

The procedure was briefly explained to the patient including the risks of contrast examination.

\section{Technique of Examination}

All patients screened before entry into the MRI scanning room for ferromagnetic objects, cardiac pacemakers, and aneurysm clips, etc. Patients were examined in the supine position on the MRI machine after proper positioning and immobilization of the head was obtained. The head coil was used for the scan. Initial topogram of the head was obtained and sequences were planned according to the MRI seizure protocol. MRI protocol at 1.5T includes the entire brain from nasion to inion, conventional routine $5 \mathrm{~mm}$ slice thickness, $\mathrm{T} 1$ and $\mathrm{T} 2$ axial sequences, $1.5 \mathrm{~mm}$ slice thickness coronal oblique. T1 weighted MPRAGE or SPGR images; $1.5 \mathrm{~mm}$ slice thickness are acquired as a 3 dimensional (3D) volume, thereby post processing and reformatting images into multiple planes. Protocol also includes coronal and axial FLAIR sequences with $2-3 \mathrm{~mm}$ slice thickness $1 \mathrm{~mm}$ inter slice gap. A conventional thin slice, T2 weighted axial and coronal sequence is obtained. Gadolinium paramagnetic contrast agent used in MRI. Contrast agent was used if a known tumour or vascular malformation. Dosage used as $0.1 \mathrm{mg} / \mathrm{kg}$ wt.

The scans were studied in detail on monitor and finally films were taken for permanent record.

\section{RESULTS}

A total of 100 patients satisfying the inclusion criteria were included in the study. The age range of patients was from neonate to elderly with male predominance, male $64(64 \%)$ and female $36(36 \%)$. GTCS was the most common clinical diagnosis constituting (80\%) cases.

\begin{tabular}{|c|c|c|}
\hline Clinical Diagnosis & $\begin{array}{c}\text { No. of } \\
\text { Patients }\end{array}$ & $\begin{array}{c}\text { Percentage } \\
\text { (\%) }\end{array}$ \\
\hline GTCS & 80 & $80 \%$ \\
\hline Myoclonic seizures & 3 & $3 \%$ \\
\hline Absence seizures & 1 & $1 \%$ \\
\hline $\begin{array}{c}\text { Simple partial } \\
\text { seizures }\end{array}$ & 7 & $7 \%$ \\
\hline $\begin{array}{c}\text { Complex partial } \\
\text { seizures }\end{array}$ & 7 & $7 \%$ \\
\hline Febrile seizures & 1 & $1 \%$ \\
\hline Motor seizures & 1 & $1 \%$ \\
\hline \multicolumn{2}{|c|}{$\begin{array}{l}\text { Table 1: Distribution of Patients } \\
\text { based on clinical diagnosis seizures }\end{array}$} \\
\hline
\end{tabular}

\begin{tabular}{|c|c|c|}
\hline MR Diagnosis & $\begin{array}{c}\text { No. of } \\
\text { Patients }\end{array}$ & Percentage \\
\hline Normal study & 35 & $35 \%$ \\
\hline Infarct with gliosis & 16 & $16 \%$ \\
\hline Infective Granuloma & 17 & $17 \%$ \\
\hline Atrophy & 1 & $1 \%$ \\
\hline $\begin{array}{l}\text { Developmental } \\
\text { malformation }\end{array}$ & 2 & $2 \%$ \\
\hline Glioma & 9 & $9 \%$ \\
\hline Meningioma & 3 & $3 \%$ \\
\hline Miscellaneous & 17 & $17 \%$ \\
\hline
\end{tabular}

\begin{tabular}{|c|c|c|c|c|c|c|c|}
\hline MR Diagnosis & <1 Years & 1-15 Years & $\mathbf{1 6 - 3 0}$ Years & $\mathbf{3 1 - 4 5}$ Years & $\mathbf{4 6 - 6 0}$ Years & $>$ 60 Years & Total \\
\hline Infarct with gliosis & 0 & 0 & 7 & 9 & 0 & 0 & 16 \\
\hline Infective Granuloma & 0 & 1 & 10 & 4 & 1 & 1 & 17 \\
\hline Atrophy & 0 & 0 & 0 & 0 & 1 & 0 & 1 \\
\hline Developmental malformation & 1 & 1 & 0 & 0 & 0 & 0 & 2 \\
\hline Glioma & 0 & 0 & 1 & 5 & 4 & 0 & 10 \\
\hline Meningioma & 0 & 0 & 0 & 0 & 3 & 0 & 3 \\
\hline Miscellaneous & 1 & 5 & 4 & 2 & 2 & 1 & 15 \\
\hline \multicolumn{7}{|c|}{ Table 3: Distribution of Abnormalities in various age groups } \\
\hline
\end{tabular}




\section{DISCUSSION}

Patients presenting with seizures can have wide range of MR imaging abnormalities depending upon the etiology. MRI can reliably identify and localize the intracranial abnormality, so that further management can be planned accordingly.

Patients presented with seizures of varying duration ranging from few days to few months. The MR examination revealed pathological findings in 65 out of 100 patients (65\%) which includes, cerebral infarct with gliosis (16\%); infective granuloma $(17 \%)$; atrophy $(1 \%)$; gliomas $(9 \%)$; cortical malformations (2\%); meningioma (3\%); other miscellaneous causes $(17 \%)$.

\section{INFECTIVE GRANULOMA}

\section{Neurocysticercosis}

Seven patients showed evidence of Neurocysticercosis (NCC). All patients had parenchymal form of NCC with multiple ring enhancing lesions in cerebral hemispheres. Lesions show T1 hypointense and T2 hyperintense contents. Few lesions showed perilesional edema. Most of lesions seen in parietal lobe and some show cystic signals with eccentric speck within the lesion. MRS showed choline peak in all patients. Five patients had single ring enhancing lesion with perilesional edema and two patients had multiple intraparenchymal lesions of different stages. Velasco TR, Zanello PA, Dalmagro $\mathrm{CL}$, et al. evaluated 512 patients of intractable epilepsy and concluded that isolated NCC was found in eight patients $(1.56 \%) .^{3}$

Tushar B. Patil, Madhuri M. Paithankar studied 40 patients with probable diagnosis of NCC and concluded that $72 \%$ patients showed one lesion, $27 \%$ with multiple lesions and common site was parietal lobe (4\%). ${ }^{4}$ Sancheti et al. in their study on NCC concluded that MRI is more sensitive in demonstration of non-calcified cerebral lesions. ${ }^{5}$

\section{Tuberculoma}

Ten patients were diagnosed as having tuberculoma on MRI scan. The lesions were well defined, rim enhancing, conglomerate with thick wall of different size. The lesions showed perilesional edema and on MRS revealed elevated lactate, lipid peak. Diagnosis of tuberculoma was based on MR appearances along with one or more following supportive evidences- H/o taking ATT in past. Any h/o contact and any e/o tuberculosis in X-ray chest.

Three patients followed after treatment and significant resolution of the lesions found and one case not reported for follow-up imaging. Naser UAMA, Abdul Ghaffur MRCP, et al. studied 925 intracranial space occupying lesions with seizures and found $1.4 \%$ intracranial tuberculoma and followed after treatment, $66.6 \%$ responded well to medical treatment and $33 \%$ failed to respond. Remarkable improvement of intracranial lesions within 6 weeks and complete resolution of the lesions within 12 weeks was found. ${ }^{6}$ Gulati et al. 1991 studied 170 children with chronic epilepsy, MRI revealed 64 tuberculomas, 27 cases of cysticercosis and 3 gliomas. ${ }^{7}$

\section{Cerebral Infarcts with Gliosis}

Sixteen patients $(16 \%)$ revealed cerebral infarction on MRI study. Six patients had venous haemorrhagic infarct in $\mathrm{B} / \mathrm{L}$ temporal lobes. One patient showed features of sub-acute hematoma in parietal lobe showing hyperintensity on T1, T2 and FLAIR sequences.
The lesion shows hypointense thin rim on T2WI, FLAIR images. Four patients showed Chronic ischemic changes with gliosis in left fronto-parietal lobe and two patients showed cystic encephalomalacia changes in left parieto-occipital lobe. Four patients showed tiny chronic ischemic lesions in deep white matter with bilateral periventricular hyperintense lesions on T2 and FLAIR with no restriction on diffusion sequences. Two patients showed old infarct with gliosis in parietal lobe region. Intracerebral haemorrhage associated with highest incidence of post stroke seizures and transient ischemic attack is associated with lowest incidence (3\%). ${ }^{8}$

\section{Cerebral Atrophy:}

One patient showed features of Cerebral atrophy.

MRI revealed atrophic changes mainly involving bilateral frontal and temporal lobes with cerebellar atrophy. Also seen were multiple old tiny ischemic areas in B/L frontoparietal region. Ghayyur Khan, et al. studied 100 patients with MRI associated symptoms of seizures, dementia and diabetes and found that cerebral atrophy in $47 \%$ male and $43 \%$ female concluded that cerebral atrophy is a complication of long standing diabetes well recognized by MRI. ${ }^{9}$

\section{Developmental Malformations}

Two patients showed the features of developmental malformations. One patient revealed findings of Corpus callosum Agenesis. Another one patient revealed absence of septum pellucidum. Jagruti P. Sanghvi, Surekha B. Rajadhyaksha and Meher Ursikar studied 76 children with seizures and CNS malformations based on MRI neuroimaging and concluded that 19 cases revealed corpus callosal dysgenesis, 9 patients lissencephaly and 9 focal cortical dysplasia, 6 pachygyria, 3 polymicrogyria, 15 tuberous scleroses and 3 Sturge-Weber syndrome.10

\section{Glioma}

Nine patients revealed evidence of glioma on MRI. Mostly, the lesions were seen in temporal lobe regions, all the lesions were single in patients.

\section{The MR Features}

The lesions hypointense on T1WI and hyperintense on both T2WI and FLAIR sequences. Mild perilesional restriction diffusion with mild mass effect seen. MRS showed elevated choline peak in most cases and the lesions showed no contrast enhancement. The above features suggestive of temporal lobe glioma and treated accordingly.

\section{Venous Sinus Thrombosis}

Four patients showed MRI features of cerebral venous sinus thrombosis in our study. Two patients showed superior sagittal sinus thrombosis and one revealed left transverse sinus thrombosis and other patient showed thrombosis in transverse and sigmoid sinuses. All patients showed hemorrhagic infarct with thrombus and one patient showed extension of thrombus into superficial cortical vein with focal gyral edema. Since all thrombus were associated with infarcts, we have considered them in one category. MRV was done in all patients. Rishi K. Gupta, et al. reported a case of Superior Sagittal Sinus (SSS) thrombosis with long history of continuous headache and MRV confirmed the presence of SSS thrombosis with small venous infarct, where CT was normal. ${ }^{11}$ 


\section{MENINGIOMA}

Three patients revealed the features of meningioma and all were convexity tumors.

In our study, the neuroimaging showed features of meningioma with mass effect.

\section{CONCLUSION}

MRI is the investigation of choice in patients with seizure disorder. The sensitivity of MRI in detecting abnormalities in patients with seizure disorder is in part associated to the underlying pathologies and by the MRI techniques and experience of the interpreting physician. Accurate diagnosis of the cause of seizure is crucial for finding an effective treatment. With its high spatial resolution, excellent inherent soft tissue contrast, multiplanar imaging capability and lack of ionizing radiation, MR imaging has emerged as a versatile tool in the evaluation of patients with seizure disorder.

MR imaging not only identifies specific epileptogenic substrates, but also determines specific treatment and predicts prognosis. Employing appropriate imaging protocols and reviewing the images in a systemic manner helps in the identification of subtle epileptogenic structural abnormalities. This study was carried out in 100 patients with clinical impression of seizures by subjecting them to magnetic resonance imaging to evaluate the spectrum of findings, various etiologic factors for seizures, and the most common imaging abnormality.

Our study concluded that infective granuloma $17 \%$, cerebral infarcts $16 \%$, atrophy $1 \%$, developmental malformation $2 \%$, gliomas $9 \%$, meningioma $3 \%$ to constitute the main etiological factors, other rare comprising SDH, SAH, ADEM, encephalitis, etc. The most common abnormality was Infective granuloma and cerebral infarct.

MR imaging is superior neuroimaging with no radiation exposure and could be the first investigation of choice in epileptic syndrome, acute cerebrovascular disease with seizure, developmental cortical malformations, and vascular malformations. Its ability in identifying subtle lesions, location, extent of the lesions and amount of findings are excellent.
Hence, we conclude that MRI plays a significant role in patients presenting with seizures with MRI seizure protocol to confirm or rule out any organic or developmental lesions.

\section{REFERENCES}

1. Vivek Gupta and Richard A Bronen. Epilepsy. In: Atlas, Scott W (eds) Magnetic Resonance Imaging of the brain and spine, $4^{\text {th }}$ edition. Philadelphia, USA: Lippincott Williams and Wilikins; 2009.p. 307-39.

2. Leslie A Hartman, MD; Sara R Nace, MD; Jane $H$ Maksimovic, DO; et al.: Epilepsy Imaging: Approaches and protocols. Applied radiology. May 2015;44(5):8-19.

3. Velasco TR, Zanello PA, Dalmagro CL, et al. evaluated 512 patients of intractable epilepsy; a cross-sectional study of 512 patients;

J Neurosurg Psychiatry. 2006 Apr; 77(4):485-8.

4. Tushar B Patil, Madhuri M Paithankar; Clinico-radiological profile and treatment outcomes in neurocysticercosis: A case study of 40 patients. Ann Trop Med Public Heath 2010; 58-63, 6.

5. Sanchetee PC, Venkataraman CS, Dhamija RM, et al. Epilepsy as a manifestation of neurocysticercosis. J Assoc Physician India 1991; 39:325-8.

6. Naser UAMA Abdul Ghaffur et al. Intracranial tuberculoma in Kuwait; Int J Tubercu Lung Dis 1998 May; 2(5):413-8, 8.

7. Gulati P, Jena A, Tripathi RP, et al. Magnetic resonance imaging in childhood epilepsy. Indian Pediatr 1991; 28:761-5, 9.

8. Myint PK, Staufenberg EFA, Sabanathan K. Post stroke seizure and post stroke epilepsy. Postgrad Med J 2006 Sept; 82(971):568-572.

9. Ghayyur Khan, Nasir Khan, Abdul Aziz. Detection of cerebral atrophy in type-II diabetes mellitus by MRI of brain; J Ayub Med Coll Abbottabad 2010; 22(2); 67-70.

10. Jagruti P Sanghvi, Surekha B Rajdhyaksha and Mehar Ursekar. Spectrum of congenital CNS malformation in pediatric epilepsy; Indian Pediatrics 2004; 41:831-838.

11. Rishi K Gupta, Aimun AB J Amjoom and Upendra $P$ Devkota. Superior sagittal sinus thrombosis presenting as a continuous headache: a case report and review of the literature; Cases Journal 2009; 2:93-61. 


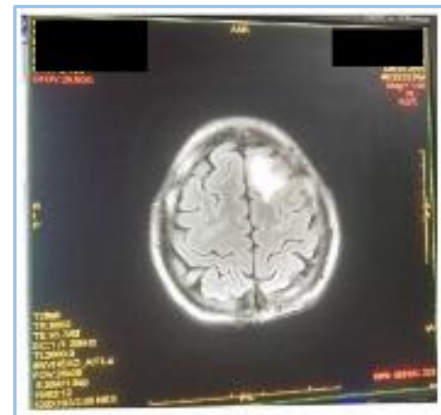

Axial T2Fl

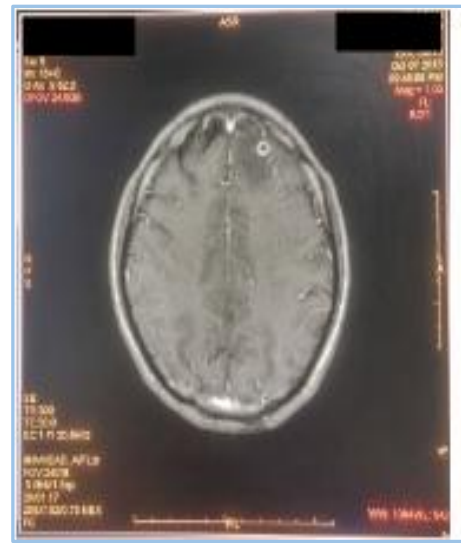

Axial T1+C

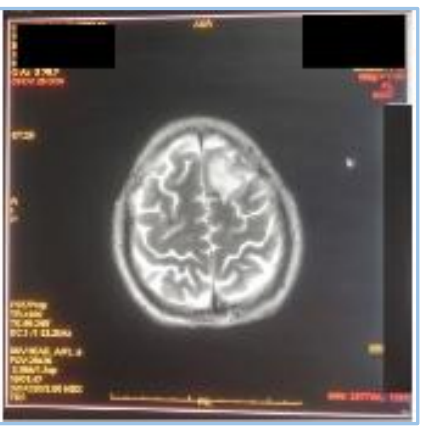

Axial T2

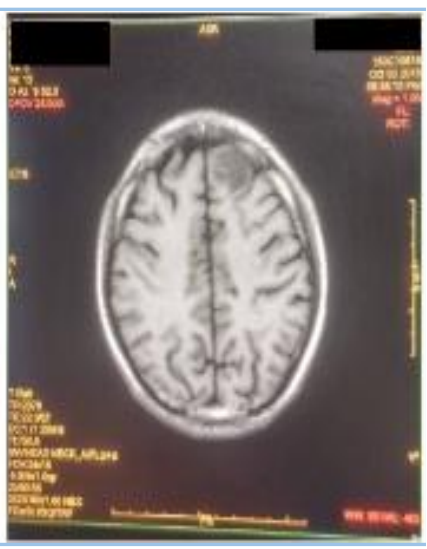

Axial T1

Fig. 1: Inflammatory Granuloma

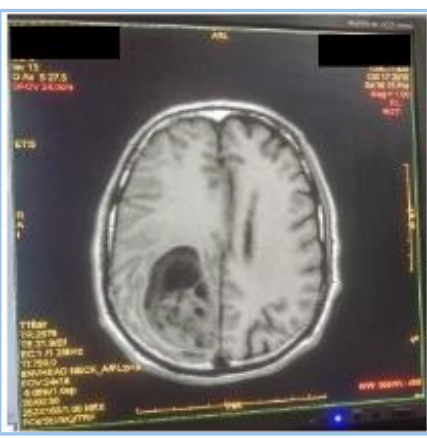

Axial T1

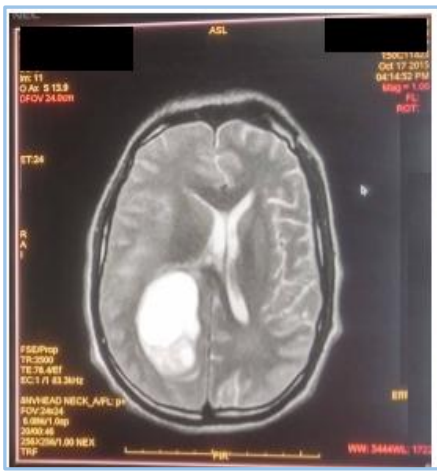

Axial T2

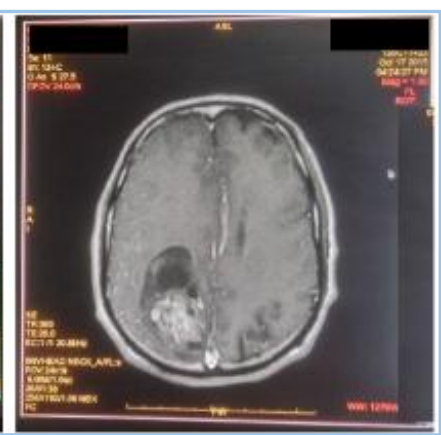

Axial T1+C

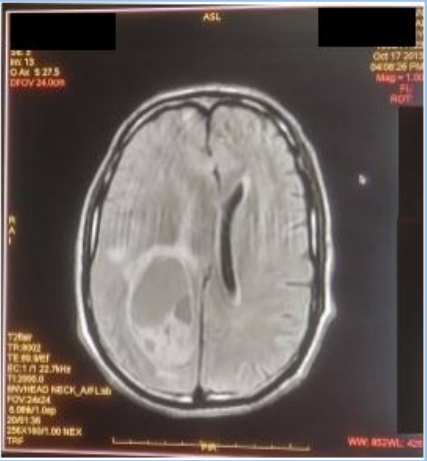

Axial T2 Fl

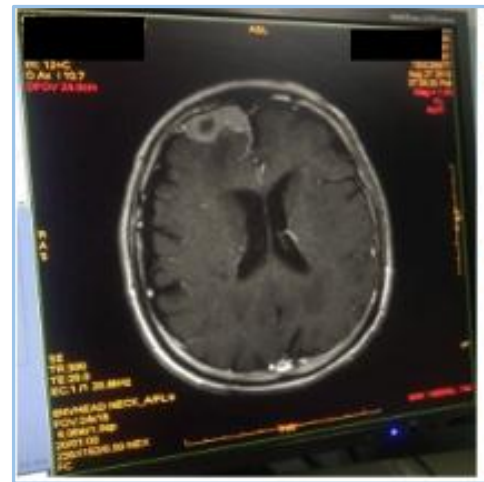

$\boldsymbol{A}$

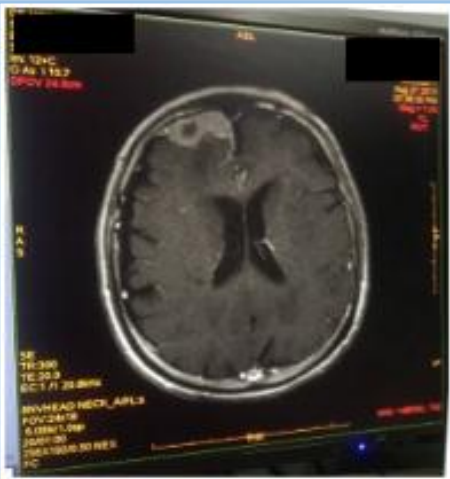

Axial T1+C

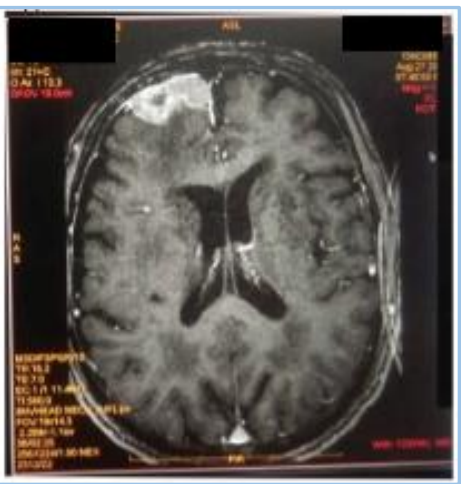

B

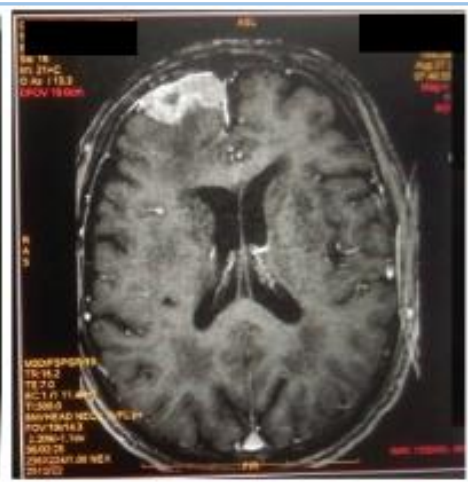

Axial T1

Fig. 3: Meningioma

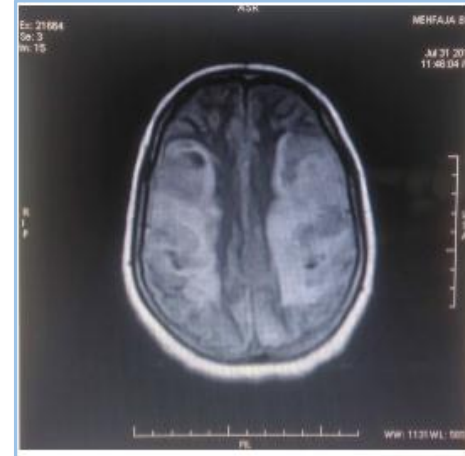

Axial T2

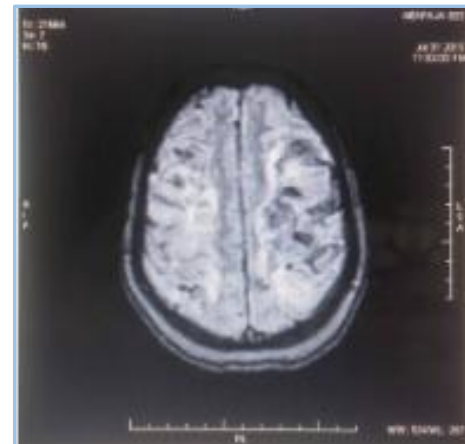

Axial T1

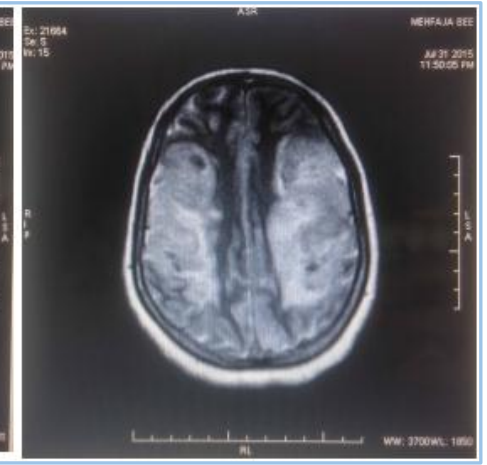

Axial T2

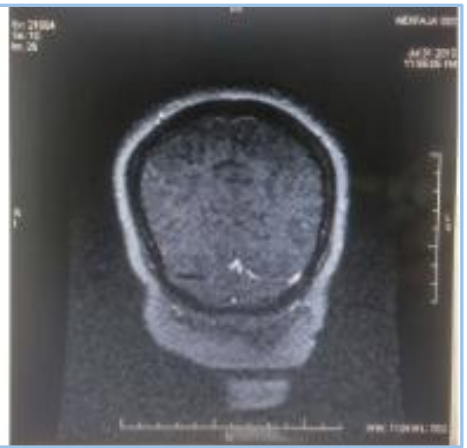

Cor T1C

Fig. 2: Glioma

Fig. 4: Venous infarct with transverse sinus thrombosis 


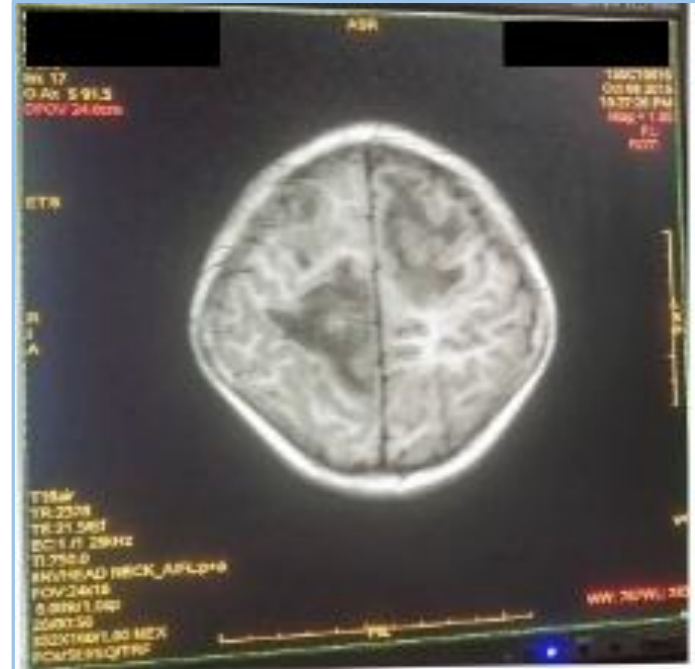

Axial T1

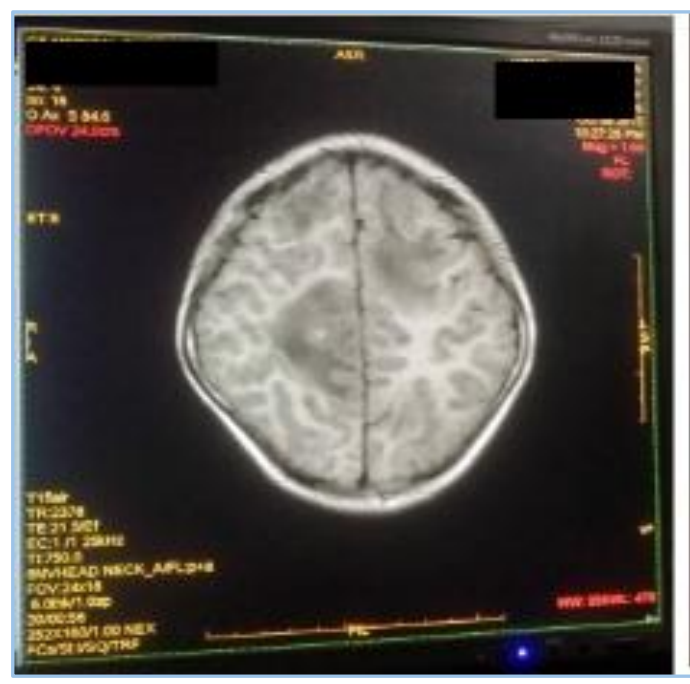

Axial T1

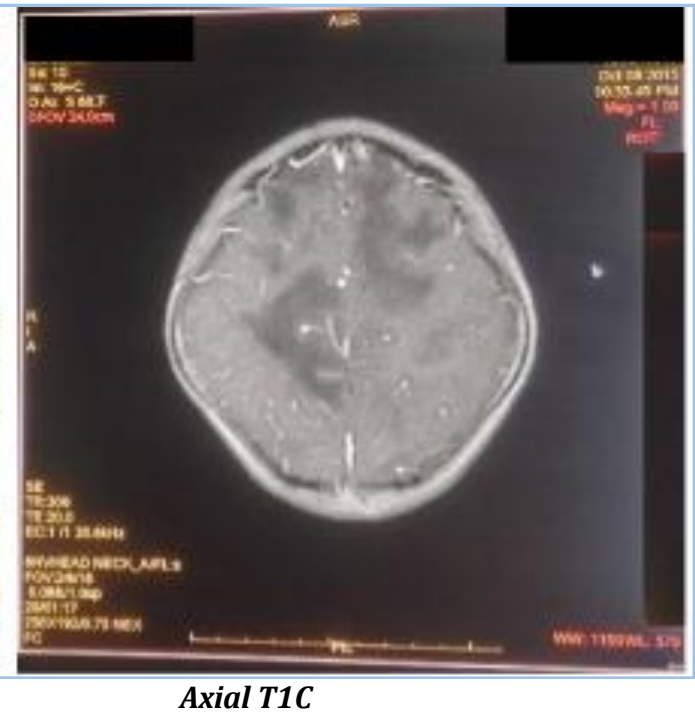

Fig. 5: Acute Demyelinating Encephalomyelitis

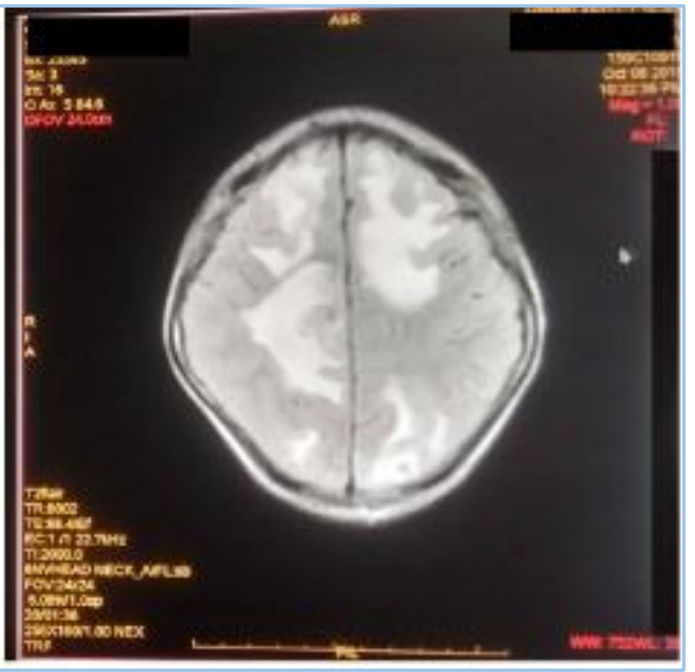

Axial T2 Fl

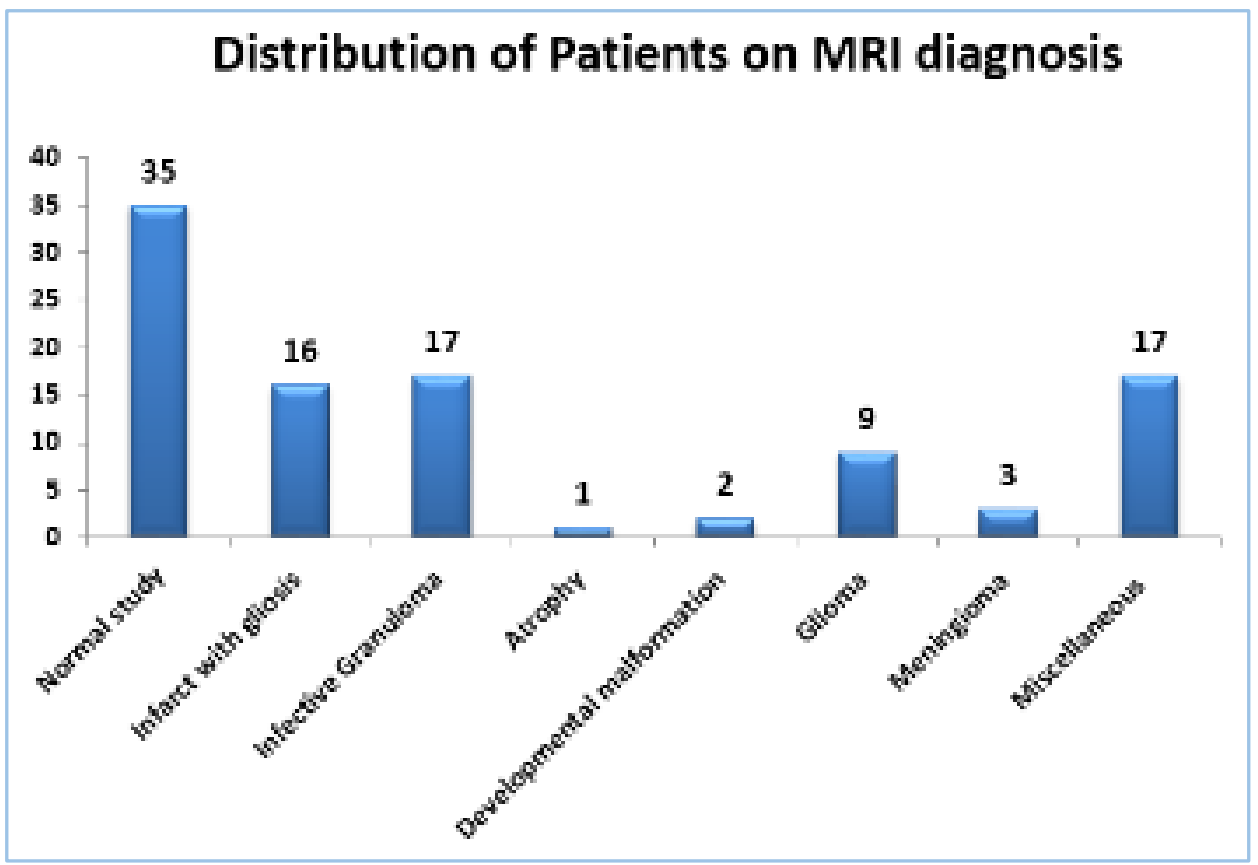

Graph 1 


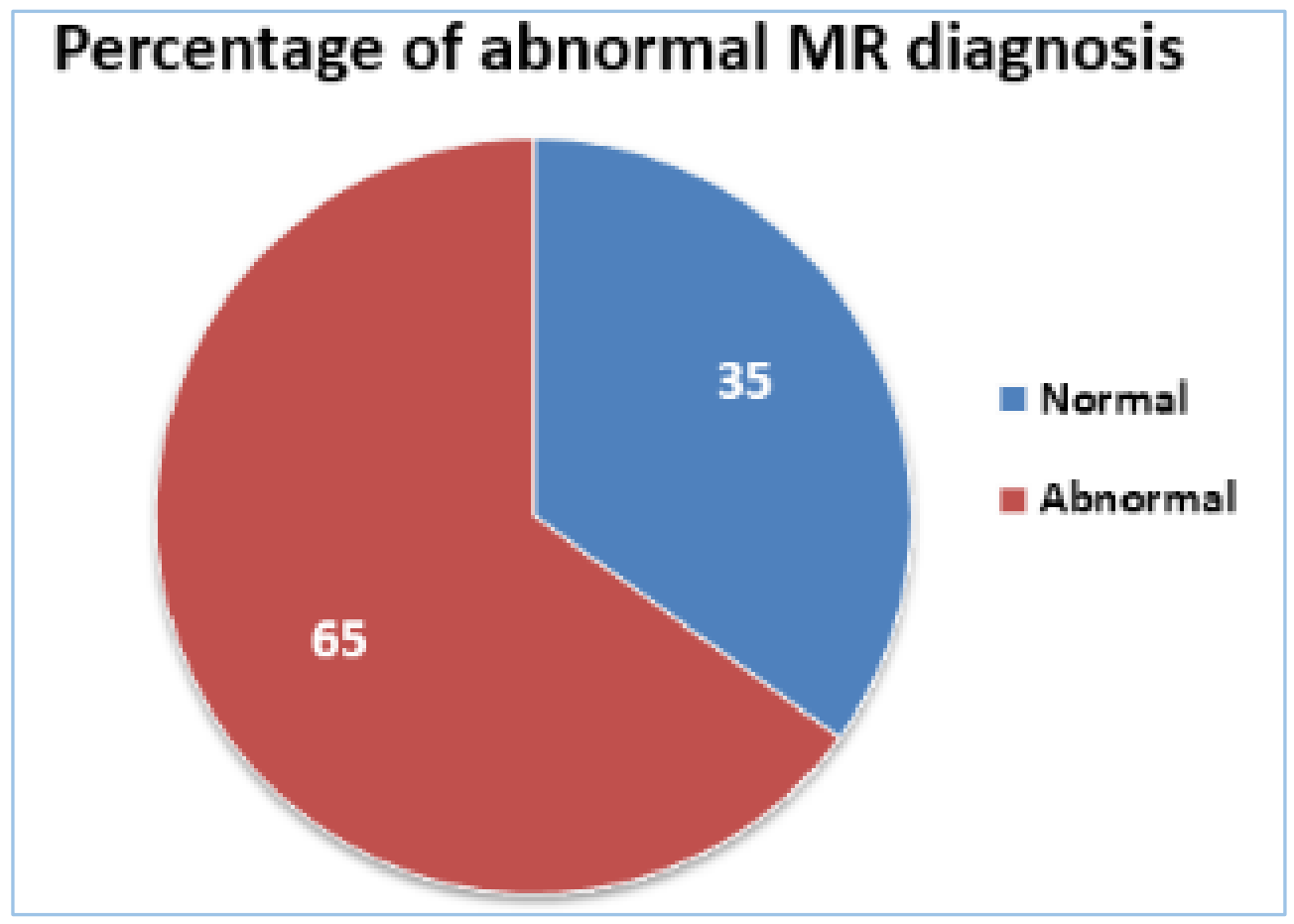

Graph 2

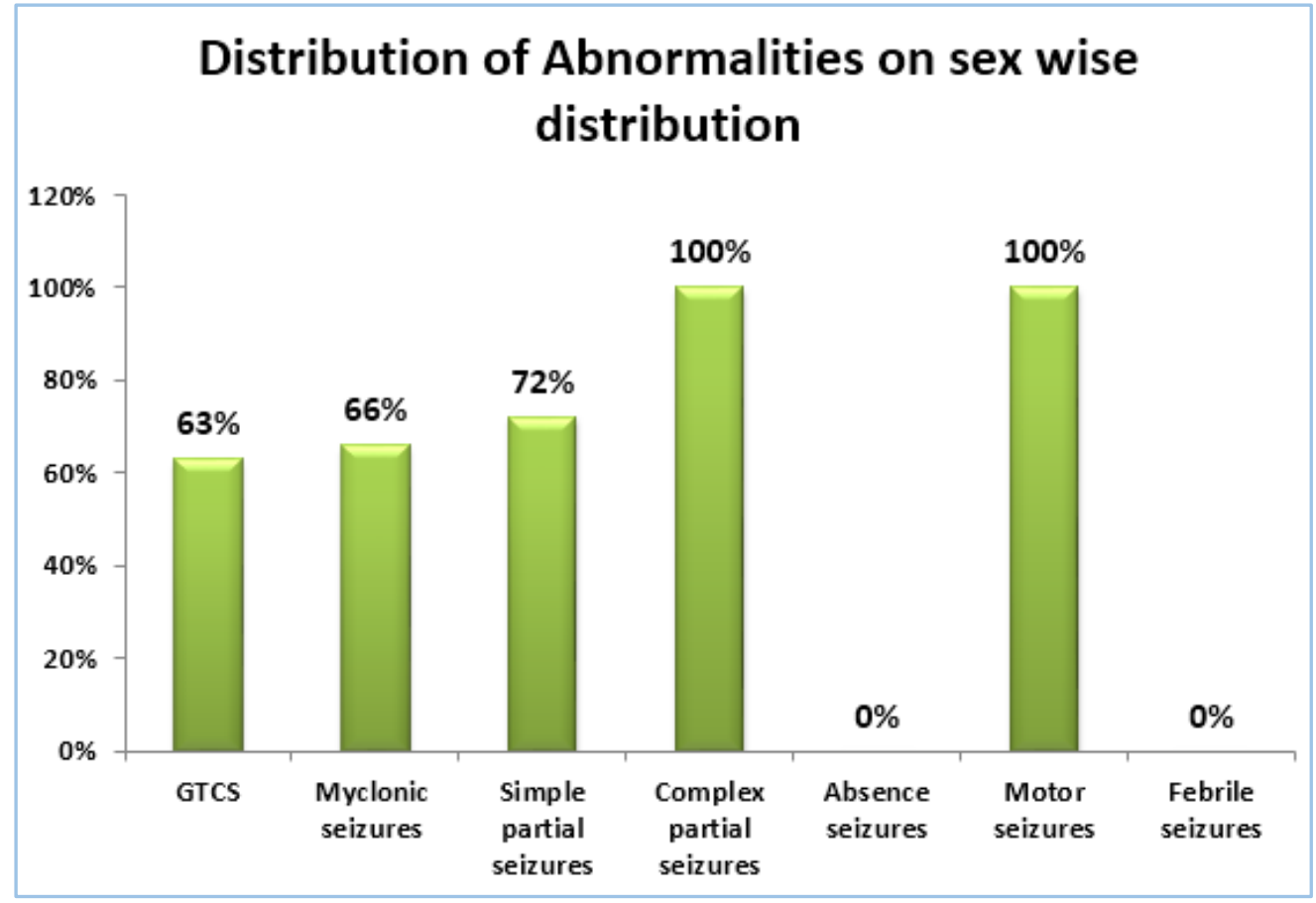

Graph 3 